\title{
Mediaciones efectuadas en el ámbito odontológico realizadas por el sistema público de salud. 2010-2015.
}

\section{Mediations in dentistry made by the public health system. 2010-2015.}

\author{
Karla Moscoso-Matus ${ }^{1 *}$, Marco Cornejo-Ovalle ${ }^{2}$, Sandra Cepeda ${ }^{3}$
}

\begin{abstract}
1. Cirujano Dentista, Especialista en Odontología Forense y Salud Publica. Facultad de Odontología, Universidad San Sebastián, Puerto Montt. Auditor del Servicio Médico Legal, Santiago de Chile. Directora Fundación Sonrisas 2. Cirujano Dentista, Especialista en Radiología Máxilo Facial, Doctor en Salud Pública, Profesor Asistente Facultad de Odontología, Universidad de Chile, Santiago de Chile. Director Fundación Sonrisas

3. Asistente Social, Magister en Gobierno y Sociedad, Encargada de Gestión de la Unidad de Mediación del Consejo Defensa del Estado, Santiago de Chile.

* Correspondencia autor: Karla Moscoso Matus Teatinos \#240, Santiago | Teléfono 228929200/ 984281855 | Email:kmoscoso@gmail.com Trabajo recibido el 12/09/2017. Aprobado para su publicación el 16/12/2017
\end{abstract}

\begin{abstract}
RESUMEN
Introducción. La Reforma de Salud del 2005 estableció la mediación como un procedimiento obligatorio para la justicia civil, con el objeto de evitar la judicialización de la salud. Esta investigación ahonda en las características de las mediaciones odontológicas del sistema público de salud en Chile.

Material y Método. Estudio transversal, de mediaciones generadas por atenciones odontológicas otorgadas en el sistema público de salud entre los años 2005 y 2015 informadas por Unidad de Mediación del Consejo de Defensa del Estado (CDE).

Resultados. No se dispone de anterior al año 2010 por falta de registro. Por tanto, solo fue factible analizar desde 2010 al 2015 . Se registran 127 mediaciones ingresadas odontológicas, el $61 \%$ reclamantes son mujeres. $60,6 \%$ proviene de la atención secundaria. $83,4 \%$ (106 casos) el reclamo es contra el establecimiento y el tratante. Sólo el $22 \%$ logra acuerdo. De estos, el $10 \%$ solo requiere explicaciones y solo el $28,5 \%$ solicita indemnización.

Conclusiones. Una mejor relación profesional-paciente disminuye el riesgo de judicialización, así como una mejor difusión del procedimiento de mediación aumentaría
\end{abstract} tasa de acuerdos.

PALABRAS CLAVES

Odontología, Mediación, Juicios en salud.

Rev. Clin. Periodoncia Implantol. Rehabil. Oral Vol. 11(1); 24-27, 2018.

\section{ABSTRACT}

Introduction. The 2005 Health Reform established mediation as a mandatory procedure for civil justice, in order to avoid prosecution of health. This research delves into the characteristics of the dental mediations of the Chilean public health system. Material and method. Cross-sectional study of mediations generated by dental care granted in the public health system between 2005 and 2015, reported by the Mediation Unit of the State Defense Council (CDE).

Results. Not available prior to 2010 due to the lack of registration. Therefore, an analysis was only feasible from 2010 to 2015 . There are 127 dentistry mediations registered, $61 \%$ of the claimants are women. $60.6 \%$ come from secondary care. In $83.4 \%$ (106 cases) the claim is against the establishment and the practitioner. Only $22 \%$ achieve agreement. Of these, $10 \%$ only require explanations and only $28.5 \%$ ask for compensation

Conclusions. A better professional-patient relationship reduces the risk of prosecution, and a better dissemination of the mediation procedure would increase agreement rate. KEYWORDS

Dentistry, Mediation, Health trials.

Rev. Clin. Periodoncia Implantol. Rehabil. Oral Vol. 11(1); 24-27, 2018.

\section{INTRODUCCIÓN}

La Reforma de Salud implementada en Chile, el año 2005, estableció la mediación como un procedimiento alternativo de resolución de conflictos derivados de la atención de salud, obligatorio para la justicia civil, con el objeto de evitar la judicialización de la práctica clínica. Entre sus características principales, está el ejecutarse a través de dos instituciones diferentes, según se trate de un caso originado por una atención en el sistema público de salud (Consejo de Defensa del Estado) o en el sistema privado de salud (Superintendencia de Salud).
A más de 10 años desde la implementación de la mediación, existe escasa información que compare ambos sistemas o que caracterice a los usuarios de la mediación, así como tampoco de las variables que pudiesen influir en ella. Esta investigación ahonda específicamente en las características de las mediaciones generadas por atenciones en el área odontológica del sistema público de salud y sus usuarios.

\section{MATERIAL Y MÉTODO}

El diseño del estudio efectuado fue un estudio de corte transversal. 
La población de estudio son los casos de mediaciones generadas por atenciones odontológicas otorgadas en el sistema público de salud entre los años 2005 y 2015.

Fuente de información: Se solicitó al jefe de la Unidad de Mediación en salud del Consejo de Defensa del Estado, la remisión de la base de datos ciega - con el fin de resguardar la confidencialidad de los casos - de mediaciones en el área odontológica, efectuadas entre los años 2005 y 2015. La base enviada corresponde a planillas Excel. En ella se explicita que con anterioridad al año 2010 , no se registraba la especialidad de las mediaciones efectuadas, por tanto, no existen los antecedentes.

Las variables analizadas fueron los casos de mediación según información sociodemográfica de los reclamantes, tipo de prestador (persona jurídica o natural), región de ocurrencia del hecho, nivel de atención en salud y tipo de establecimiento donde ocurrió el hecho, tipo de daño reclamado, resultado de la mediación (con o sin acuerdo), tipo de reparación solicitada, montos involucrados en indemnizaciones.

Los datos fueron analizados utilizando el software estadístico SPSS V 2.0 (IBM, NY, USA) y el programa Excel 2013 (Microsoft Office, NY, USA).

Estos autores informan que una de sus coautores ejerce sus funciones en la Unidad del CDE que otorgó la información para este estudio.

\section{RESULTADOS}

Para el periodo 2005-2009, no se dispone de información ya que la entidad no cuenta con datos registrados para esos años. Desde el año 2010-2015, se registran 127 mediaciones ingresadas, cuya "especialidad médica" involucrada es odontología. El $61 \%$ de los reclamantes corresponde a mujeres. El detalle de la distribución según año y sexo del reclamante se observa en el gráfico $\mathrm{N}^{\circ} 1$.

Las características sociodemográficas de los usuarios reclamantes se observan en la tabla $\mathrm{N}^{\circ} 1$.

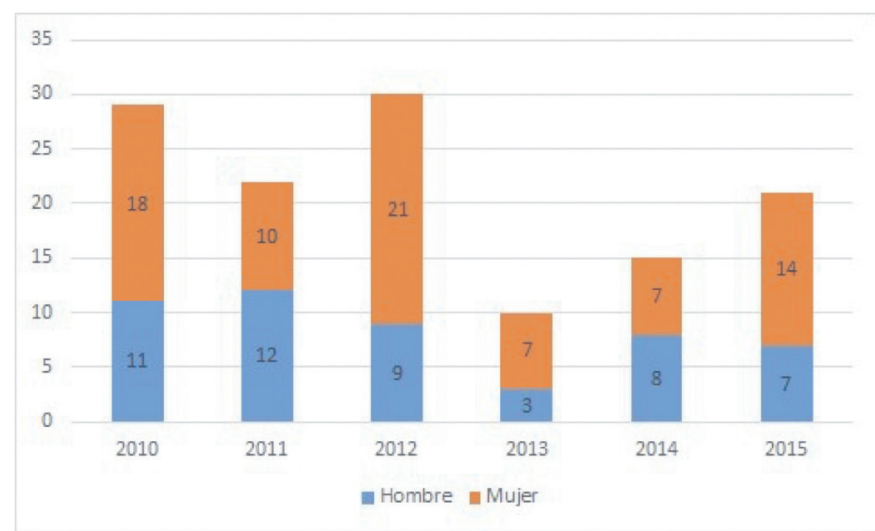

Gráfico 1. Distribución de los casos de mediaciones públicas odontológicas a ingresados al CDE según año y sexo del reclamante. Años 2010-2015.

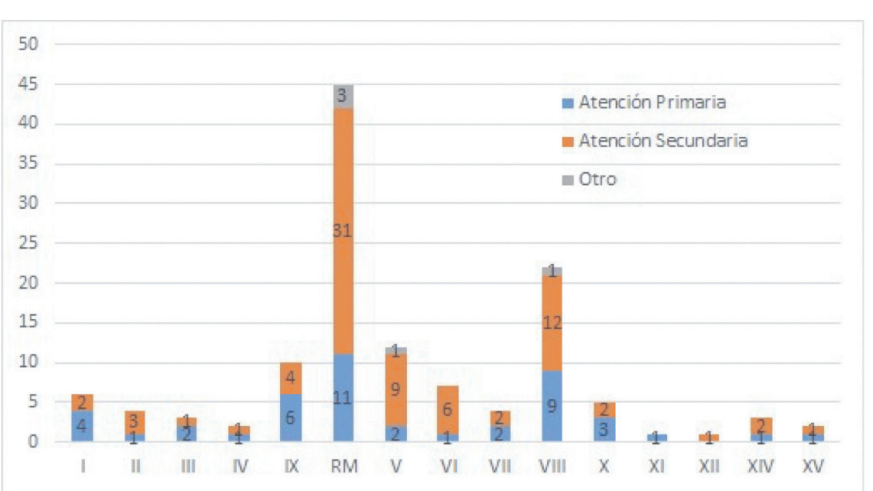

Gráfico 2. Distribución de las mediaciones públicas odontológicas según región de ocurrencia y nivel de atención de salud. Años 2010-2015.

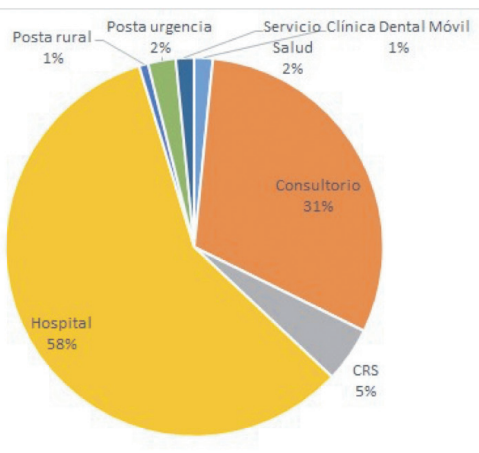

Gráfico 3. Distribución de las mediaciones públicas odontológicas según tipo de establecimiento involucrado en el hecho. Años 2010-2015.

hospitalarias. (grafico $\mathrm{N}^{\circ} 3$ )

En relación al tipo de establecimiento involucrado en la mediación, se observa que la mayoría de los casos (58\%) corresponde a atenciones efectuadas en hospital y/o CRS.

La distribución de los casos de mediaciones odontológicas según tipo de establecimiento involucrado y nivel de atención se observa en la tabla $\mathrm{N}^{\circ} 2$.

De los casos analizados, en el 83,4\% (106 casos) el reclamo se efectuó contra el establecimiento y, además, contra el odontólogo tratante

Respecto al resultado de la mediación (tabla $\mathrm{N}^{\circ} 3$ ), sólo el $22 \%$ logra acuerdo (28 casos) y un $65 \%$ finaliza con falta de acuerdo (82 casos). El porcentaje restante finaliza por causales administrativas (inasistencia,

Tabla 1. Distribución de las mediaciones públicas odontológicas según sexo, edad, nivel educacional y ocupación de los reclamantes. Años $2010-2015$.

\begin{tabular}{|c|c|c|c|c|c|}
\hline SEXO & $\mathrm{N}^{\circ}$ casos & edad & ds & Nivel educacional $^{a}$ & Ocupación \\
\hline Masculino & 50 & 35,06 & 19,01 & \multirow{3}{*}{$\begin{array}{c}\text { - 7,09\% educación universitaria/técnica superior completa y } 4,72 \% \\
\text { incompleta } \\
-7,09 \% \text { educación básica completa y } 6,30 \% \text { incompleta } \\
-18,11 \% \text { educación media completa y } 9,45 \% \text { incompleta } \\
-2,36 \% \text { sin educación formal }\end{array}$} & \multirow{3}{*}{$\begin{array}{c}2,36 \% \text { desempleado } \\
4,72 \% \text { jubilado } \\
7,87 \% \text { trabajador independiente } \\
22,05 \% \text { estudiante } \\
25,20 \% \text { labores del hogar } \\
29,92 \% \text { trabajador dependient }\end{array}$} \\
\hline Femenino & 77 & 37,68 & 17,0 & & \\
\hline Total & 127 & 36,65 & 17,79 & & \\
\hline
\end{tabular}

ds: desviación estándar

a de los registros el 44,88 \% no posee antecedentes de nivel de educación y el 7,87\% de ocupación

Respecto al origen de las mediaciones según nivel de atención, se observa que el $60,6 \%$ proviene de la atención secundaria de salud, en tanto, el $35,4 \%$ se genera por atenciones realizadas en establecimientos del nivel de atención primaria de salud.

En relación al origen geográfico de los casos de mediaciones odontológicas, este se observa en el gráfico $\mathrm{N}^{\circ} 2$.

En relación al tipo de establecimiento involucrado en la mediación, se observa que la mayoría de los casos $(58 \%)$ son generadas por atenciones plazo vencido, etc.)

De los casos que finalizan con acuerdo (gráfico $\mathrm{N}^{\circ} 4$ ), el $82 \%$ solicita prestaciones asistenciales, solas o asociadas a otro tipo de compensación (explicaciones, disculpas o indemnización), el 10\% solo requiere explicaciones y solo el $28,5 \%$ solicita indemnización (sola o asociada a otro tipo de compensación).

En relación a las denuncias según los daños reclamados, la mayoría de estos corresponden a reclamos por daño psicológico (gráfico $N^{\circ} 5$ ). 
Tabla 2. Distribución neta y porcentual de las mediaciones públicas odontológicas según tipo establecimiento de salud de origen y nivel de atención. Años 2010-2015.

\begin{tabular}{|c|c|c|c|c|c|c|c|c|c|c|c|c|c|c|c|c|}
\hline \multirow{3}{*}{$\begin{array}{l}\begin{array}{l}\text { Nivel de } \\
\text { atención }\end{array} \\
\begin{array}{l}\text { Atención } \\
\text { Primaria }\end{array}\end{array}$} & \multicolumn{16}{|c|}{ Tipo de establecimiento } \\
\hline & \multicolumn{2}{|c|}{$\begin{array}{c}\text { Clínica } \\
\text { Dental Móvil }\end{array}$} & \multicolumn{2}{|c|}{ Consultorio } & \multicolumn{2}{|c|}{ CRS } & \multicolumn{2}{|c|}{ Hospital } & \multicolumn{2}{|c|}{$\begin{array}{l}\text { Posta } \\
\text { rural }\end{array}$} & \multicolumn{2}{|c|}{ Posta urgencia } & \multicolumn{2}{|c|}{ Servicio Salud } & \multicolumn{2}{|c|}{ Total general } \\
\hline & 2 & $4 \%$ & 39 & $87 \%$ & 0 & $0 \%$ & 0 & $0 \%$ & 1 & $2 \%$ & 3 & $7 \%$ & 0 & $0 \%$ & 45 & $35 \%$ \\
\hline $\begin{array}{l}\text { Atención } \\
\text { Secundaria }\end{array}$ & 0 & $0 \%$ & 0 & $0 \%$ & 5 & $6 \%$ & 72 & $94 \%$ & 0 & $0 \%$ & 0 & $0 \%$ & 0 & $0 \%$ & 77 & $61 \%$ \\
\hline Otro & 0 & $0 \%$ & 0 & $0 \%$ & 1 & $20 \%$ & 2 & $40 \%$ & 0 & $0 \%$ & 0 & $0 \%$ & 2 & $40 \%$ & 5 & $4 \%$ \\
\hline Total general & 2 & $2 \%$ & 39 & $31 \%$ & 6 & $5 \%$ & 74 & $58 \%$ & 1 & $1 \%$ & 3 & $2 \%$ & 2 & $2 \%$ & 127 & $100 \%$ \\
\hline
\end{tabular}

Tabla 3. Distribución neta de las mediaciones públicas odontológicas, según tipo de termino. Años 2010-2015.

\begin{tabular}{|c|c|c|c|c|c|c|c|c|c|c|c|c|c|c|}
\hline \multirow{2}{*}{ Tipo de Termino } & \multicolumn{14}{|c|}{ Año ingreso } \\
\hline & \multicolumn{2}{|c|}{2010} & \multicolumn{2}{|c|}{2011} & \multicolumn{2}{|c|}{2012} & \multicolumn{2}{|c|}{2013} & \multicolumn{2}{|c|}{2014} & \multicolumn{2}{|c|}{2015} & \multicolumn{2}{|c|}{ Total general } \\
\hline Acuerdo & 3 & $10,3 \%$ & 5 & $22,7 \%$ & 10 & $33,3 \%$ & 3 & $30,0 \%$ & 5 & $33,3 \%$ & 2 & $9,5 \%$ & 28 & $22,0 \%$ \\
\hline Falta de acuerdo & 19 & $65,5 \%$ & 14 & $63,6 \%$ & 15 & $50,0 \%$ & 5 & $50,0 \%$ & 10 & $66,7 \%$ & 19 & $90,5 \%$ & 82 & $64,6 \%$ \\
\hline $\begin{array}{l}\text { Inasistencia de una o } \\
\text { ambas partes }\end{array}$ & 2 & $6,9 \%$ & 1 & $4,5 \%$ & 0 & $0,0 \%$ & 0 & $0,0 \%$ & 0 & $0,0 \%$ & 0 & $0,0 \%$ & 3 & $2,4 \%$ \\
\hline Otra causa & 0 & $0,0 \%$ & 0 & $0,0 \%$ & 1 & $3,3 \%$ & 1 & $10,0 \%$ & 0 & $0,0 \%$ & 0 & $0,0 \%$ & 2 & $1,6 \%$ \\
\hline Vencimiento del plazo & 5 & $17,2 \%$ & 2 & $9,1 \%$ & 4 & $13,3 \%$ & 1 & $10,0 \%$ & 0 & $0,0 \%$ & 0 & $0,0 \%$ & 12 & $9,4 \%$ \\
\hline Total & 29 & $100,0 \%$ & 22 & $100,0 \%$ & 30 & $100,0 \%$ & 10 & $100,0 \%$ & 15 & $100,0 \%$ & 21 & $100,0 \%$ & 127 & $100,0 \%$ \\
\hline
\end{tabular}

\section{DISCUSIÓN}

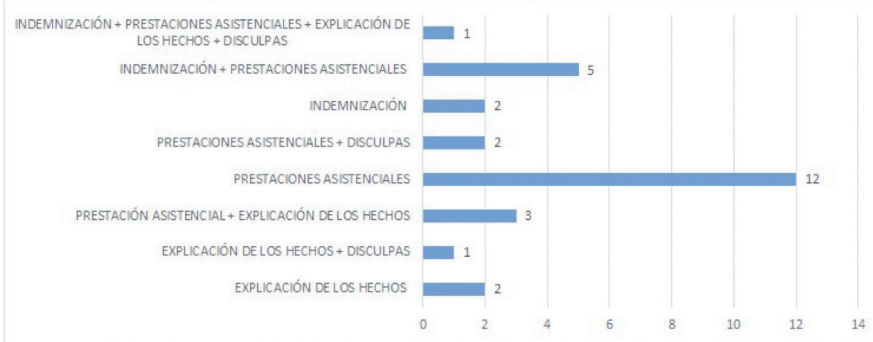

Gráfico 4. Distribución neta de las mediaciones públicas odontológicas, finalizadas con acuerdo, según tipo de reparación pactada. Años 2010-2015

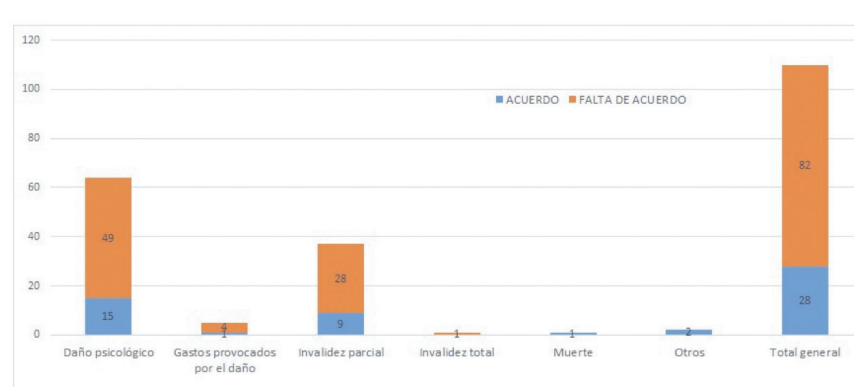

Gráfico 5. Distribución de las mediaciones públicas odontológicas según daño reclamado y tipo de acuerdo (con acuerdo y sin acuerdo). Años 2010-2015.

Respecto del tipo de daño que genera una mediación, se observa que, descontando los casos finalizados por causas administrativas, el $59 \%$ de los casos sin acuerdo y el $53 \%$ de los con acuerdo, argumentan daño psicológico.
En este estudio se caracterizan las mediaciones generadas por atenciones en el área odontológica del sistema público de salud y sus usuarios, desde 2010 a 2015.

Respecto a las mediaciones referidas, se debe considerar cuales son las razones por las cuales un paciente o su familia inician un proceso legal contra su profesional tratante. Lee ${ }^{(1)}$ citando a Beckman identifica a la falta de disponibilidad de profesionales, preocupaciones del paciente o la familia, la falta de información, la falta de comprensión y la falta de cuidado y/o colaboración en la entrega de la atención, en suma, la mayoría son atribuidas al fracaso de la comunicación entre el profesional de salud y el paciente ${ }^{(1,2,3)}$. Esto resulta gravitante pues la literatura afirma fehacientemente que gran parte de los pacientes solo buscan una explicación honesta ${ }^{(1,2,4,5,5)}$. Ante lo que es una repuesta tan simple a un problema tan complejo, es que resulta relevante dimensionar los volúmenes de litigios en salud a que nos enfrentamos. Tradicionalmente se habla de un crecimiento explosivo de los casos odontológicos judicializados. Zanin7 explícita que en Brasil los casos se incrementaron un $380 \%$ entre el año 2000 y el 2011. En nuestro país, el CDE indica que entre los años 2005 y 2015 , de las 6.926 solicitudes de mediación informadas, el $1,8 \%$ son de origen odontológico $(n=127)$, con un peak de 30 casos el año 2012, tres veces más que los generados el año 2013. Estos autores no identifican causales de estas variaciones.

De lo anterior, es necesario contextualizar que este bajo número de casos contrasta con el alto porcentaje de población bajo cobertura de aseguramiento que posee el sistema público en todas las áreas de la salud, cercano al $70 \%$ del total del país, pero que otorga un reducido número de atenciones odontológicas, que no sobrepasa el $6 \%$ de cobertura, y con el alto porcentaje de incumplimiento de la lex artis de los odontólogos en los casos que son judicializados ${ }^{(8)}$. Algunas explicaciones a este hecho podrían corresponder a debilidades en los registros del CDE y/o al grado de detalle de estos. Sin embargo, la respuesta más plausible es que mayoritariamente las atenciones dentales son ejecutadas por el sistema privado de salud y por tanto el grueso de las mediaciones odontológicas debiesen generarse en el sistema privado de salud.

En nuestro estudio, el $60,6 \%$ de los casos de mediaciones públicas odontológicas corresponde a mujeres, similar a lo observando por Zanin $(72 \%)$. Este resultado que está acorde a las mayores tasas de uso de servicios sanitarios por parte de estas y a lo reportado por Portela ${ }^{(9)}$ en su estudio de mediaciones odontológicas privadas. Ese mismo autor estimó que el 29\% de los reclamos eran contra el profesional, $35 \%$ solo a la institución y $26 \%$ a 
ambos. En esta oportunidad, ante nuestra solicitud de información, el Consejo de Defensa del Estado no se mencionan antecedentes donde se denuncie solo al establecimiento, pero si cuando éstas son contra el establecimiento y/o el profesional. Es así que sabemos que el $14,5 \%$ de los casos fue solo contra el profesional y el $83,4 \%$ es contra, a lo menos, un establecimiento y un profesional. Al respecto, cabe hacer presente que, en el sistema público de salud, la eventual responsabilidad por falta de servicio corresponde al Estado y a las personas, cuando las faltas son individuales.

Resulta interesante que el grueso de los reclamos se concentre, según nivel de atención, en la atención odontológica secundaria (61\%), predominando ampliamente, según tipo de establecimiento, los hospitales (58\%) frente a los Centros de Referencia (CRS); pues la cobertura de este nivel de atención es aún más escasa ${ }^{(11)}$ tanto por aspectos propios del tipo de atenciones como por la falta de profesionales que deben otorgarlas. El antecedente anterior debe ser analizado considerando varios aspectos. Primeramente, que existe fuerte evidencia que indica que la judicialización de la atención no está asociada a la existencia de daño, es más, según un estudio efectuado el 2004, Studdert ${ }^{(10)}$ sobre 21.000 fichas médicas, determinó que $4,6 \%$ de las atenciones hospitalarias mostraban injurias iatrogénicas, pero sólo el $2 \%$ de negligencias dieron lugar a reclamaciones. En segundo lugar, se debe mencionar que con la promulgación de la Ley $N^{\circ} 20.584$ de derechos y deberes de los pacientes, se volvió obligatorio que los establecimientos de salud poseen oficinas de reclamo. Al respecto, un estudio realizado por la Universidad de Valparaíso(12) estima que tras la revisión de la totalidad de reclamos odontológicos (446) generados en uno de los 29 servicios de salud del país (Valparaíso-San Antonio), entre los años 2008 a julio 2012 y en virtud de la Ley N ${ }^{\circ} 19.880$ de participación ciudadana. Esos reclamos a su vez representan aproximadamente el 4,96\% de la totalidad de reclamos de salud recibidos en ese mismo periodo de tiempo y lugar. En paralelo, en el análisis de este mismo estudio, no puede dejar de mencionarse que $8 \%$ de los requerimientos correspondieron a solicitudes de información y $35 \%$ a felicitaciones y que, entre los reclamos odontológicos, el $55 \%$ tiene orígenes administrativos contra $43 \%$ clínicos en la atención primaria, cambiando a $18 \%$ administrativos contra $49 \%$ clínicos en la atención secundaria. Por tanto, resulta de interés analizar la relación entre reclamos y la atención secundaria observada en las mediaciones efectuadas por el Consejo de Defensa del Estado y los datos observados por Raby en sus tesis ${ }^{(12)}$. En ambos casos se puede suponer que una adecuada gestión del riesgo sanitario podría significar una mejora en la atención, lo que se lograría implementando estrategias de prevención a nivel odontológico hospitalario, incluyendo capacitación en estos temas, manejo y mantención de la relación profesionalpaciente ${ }^{(13)}$, así como el manejo de crisis ${ }^{(14)}$.

De las 127 mediaciones odontológicas efectuadas, el 59,8\% argumenta daño psicológico y el $31,4 \%$ invalidez parcial. Digno de ser mencionado es que en 2 casos $(1,53 \%)$ la causal de la mediación, fue la muerte del paciente. Esto nos enfrenta al paradigma de la inocuidad de la atención dental. A lo anterior debe agregarse que según Moscoso(8), del total de pericias médico legales odontológicos, el porcentaje mayoritario fue originado por causales quirúrgicas. En base a ello, tal vez sea factible cuestionar el perfil de egreso, la calidad y/o ética de los profesionales que egresan en el país. Seria por tanto interesante determinar la existencia de una relación entre años de egreso y casuística de fallas.

En Chile se estima que las demandas que debió enfrentar el sector público de salud entre los años 2001 y 2002 ascenderían a la suma de US\$ 40 a 50 millones por año(15). Sin embargo, contrario a lo señalado por Portela (2013), quien reporta que $35 \%$ requieren indemnización y de los cuales el $32 \%$ requiere además prestaciones asistenciales, en nuestro estudio, el $82,1 \%$ de los casos finalizados con acuerdo sólo requiere prestaciones asistenciales y únicamente el $28,5 \%$ busca indemnización monetaria, sola $(7,1 \%)$ o asociada a otras condiciones $(21,4 \%)$. Al mismo tiempo, es de interés analizar más profundamente porqué solo el $25,4 \%$ de las 110 mediaciones finalizadas logra llegar acuerdo y los factores que pudiesen influenciando estas cifras. En opinión de esos autores uno de los factores que podrían afectar es una socialización poco profusa de la mediación en salud, como mecanismo alternativo de solución de controversias. Lo anterior conlleva al desconocimiento de sus ventajas, tales como flexibilidad, menores costos, etc., y por tanto a su desaprovechamiento. Urge por tanto aumentar la difusión de estas materias entre el público general, así como la sensibilización de los abogados de los stakeholders.

Al finalizar, quisiéramos destacar que la mala praxis no solo tiene consecuencias en el usuario de la atención, quien manifiesta su disconformidad a través de un reclamo, mediación y/o juicio, también posee consecuencias en los profesionales, describiéndose "que los profesionales demandados sufren de diferentes grados de perturbación emocional: vergüenza, temor, duda, aislamiento, dificultad para concentrarse, etc., independientemente de si creen que un evento médico adverso se debe a su error (...), el estrés psicológico asociado con el litigio puede dañar irreversiblemente la vida profesional (...)"1(1). Por tanto, es factible asegurar que la judicialización de la salud no es gratuita para nadie.

\section{CONCLUSIONES}

En general las mediaciones son atribuibles al fracaso de la comunicación entre el profesional de salud y el paciente. En nuestro país, las mediaciones odontológicas públicas efectuadas entre los años 2005 y 2015 son 127 y corresponden al 1,8\% del total de mediaciones efectuadas por el Consejo de Defensa del Estado, contrastando con la alta cobertura de aseguramiento que posee el sistema público de salud en el país. El $60,6 \%$ de los casos corresponde a mujeres, el $14,5 \%$ de los casos fue solo contra el profesional y el $83,4 \%$ es contra, a lo menos, un establecimiento y un profesional. El grueso de los reclamos se concentra en la atención odontológica secundaria $(61 \%)$, predominando los hospitales (58\%) frente a los Centros de Referencia (CRS). El 59,8\% de los casos argumenta daño psicológico y el 31,4\% invalidez parcial, logrando acuerdo entre las partes solo el $24,5 \%$. Todo indica que una mejor relación profesional-paciente disminuye el riesgo de judicialización, así como una mejor difusión del procedimiento de mediación aumentaría su tasa de acuerdos.

\section{FUENTES DE APOYO FINANCIERO}

Sin fuentes de apoyo financiero.

\section{CONFLICTO DE INTERÉS}

Los antecedentes aportados por el CDE fueron elaborados por la Unidad de Mediación en salud de esa repartición, donde labora uno de los co-autores.

\section{RELEVANCIA CLÍNICA}

A más de 10 años desde la implementación de la mediación, existe escasa información que compare los resultados obtenidos entre el Consejo de Defensa del estado y la Superintendencia de Salud, así como la caracterización de los usuarios de la mediación y las variables que pudiesen influir en ella. Por tanto, este estudio es un gran aporte en general al sistema de resolución de conflicto por vías no judiciales, pero también al área de salud.

\section{Bibliografía}

1. Lee $D$ and Lai P. The practice of mediation to resolve clinical, bioethical, and medical malpractice disputes. Hong Kong Med J. 2015; 21:560-4.

2. Elango S. Medical Negligence suits: risk management. Med J Malaysia. 2003;58 (4):625-630

3. Fong $\mathrm{Ha} \mathrm{J}$ and Longnecker $\mathrm{N}$. Doctor-patient communication: a review. Ochsner $\mathrm{J}$. 2010;10 (1):38-43.

4. Sohn D, Sonny B. Medical malpractice reform: The role of alternative dispute resolution. Clin Orthop Relat Res. 2012; 470:1370-8.

5. Brown H, Simanowitz A. Alternative dispute resolution and mediation. Qual Health Care $1995 ; 4: 151-8$

6. Stern $\mathrm{C}$ and Schechter $\mathrm{C}$. Mediating medical malpractice lawsuits gainst Hospitals: New York city's pilot project. Health Aff (Millwood). 2006;25(5):1394-1399.

7. Zanin A, Herrera M, Melani R. Civil liability: characterization of the demand for lawsuits against dentists. Braz Oral Res. 2016;30(1): e91.

8. Moscoso $\mathrm{K}$ and Smok, P. Responsabilidad sanitaria en odontólogos. Casuística de casos evaluados en el Servicio Médico Legal de Chile. Rev Med Chile.2015;143:345-351.

9. Portela F. Caracterización de los aspectos bioéticos en los reclamos realizados ante la Superintendencia de Salud de Chile en el área odontológica. Acta Bioeth. 2013; 19:143-52
10. Studdert D, Mello M and Brennan T. Medical malpractice. N Engl J Med. 2004; 350:283-292.

11. Leiva C, Llach E. Proceso de atención ambulatoria nivel secundario y terciario. [on line]. Ministerial de Salud. [citado el 03 de mayo de 2017]. Disponible en http:// web.minsal.cl/wp-content/uploads/2016/09/8_LINEA-DE-TRABAJO-PROCESOASISTENCIAL-DE-ATENCION-AMBULATORIA.pdf

12. Raby I, Selame M. Responsabilidad profesional odontológica en servicio de Salud Valparaíso San Antonio, parte I. [Tesis para optar al Título de Cirujano Dentista]. Universidad de Valparaíso. Facultad de Odontología. 2013 [Acceso 28.08.2017] Disponible en http://bibliotecas.uv.cl/2013

13. Overton A, Lowry A. Conflict management: Difficult conversations with difficult people. Clin Colon Rectal Surg; 2013; 26:259-264.

14. Bridget B, Kinney J, Fitzgerald M; Murdoch-Kinch C; Guenther M, Ridley R, Whitman L, Ramaswamy V. Perceptions of a standardized patient instructor conflict resolution program. J Dental Educ.2014;78(10): 1387-04.

15. Rencoret G. Auditorías médicas: demandas y responsabilidad por negligencias médicas. Gestión de calidad: riesgos y conflictos. Rev Chil Radiol. 2013;9(3): 157-160 